\title{
A utilização de organizadores prévios para o ensino de estequiometria: uma proposta de unidade de ensino potencialmente
}

significativa

Franciele Tatiana Haupt*, Daniele Trajano Raupp**, Vladimir Lavayen ${ }^{\star \star \star}$

\section{Resumo}

Este trabalho apresenta uma proposta didática para o ensino de estequiometria com base na teoria da aprendizagem significativa e organizada no formato de uma unidade de ensino potencialmente significativa. $O$ formato da sequência de aulas visa a promover um aprendizado de qualidade, que difere do aprendizado mecânico, tendo em conta que o conhecimento prévio dos alunos influencia fortemente a aprendizagem. Situações e problemas que envolvem o relacionamento com ingredientes e receitas culinárias são utilizados aqui para agregar novos conhecimentos. A justificativa para o desenvolvimento dessa estratégia de ensino é que a estequiometria é identificada como um assunto desafiador devido à necessidade do uso e da interpretação de linguagens químicas, físicas e matemáticas. Como resultado, espera-se que esta unidade contribua para a melhor compreensão dos conceitos de estequiometria e promova o uso de materiais de aprendizagem potencialmente significativos, além de demonstrar a construção de relações lógicas com o conhecimento científico e o distanciamento do ensino de química com base apenas na memorização de conteúdos.

Palavras-chave: ensino de química; aprendizagem significativa; conhecimento prévio; cálculo estequiométrico; reagente limitante.

* Licencianda em Química da Universidade Federal do Rio Grande do Sul. Brasil. E-mail fran.haupt@hotmail.com

** Doutora em Educação em Ciências Docente do Instituto de Química e dos Programa de Pós-Graduação em Educação em Ciências e PROFQUI da Universidade Federal do Rio Grande do Sul. Brasil. E-mail daniele. raupp@ufrgs.br

*** Doutor em Química. Docente do Instituto de Química e do Programa de Pós-Graduação em Química da Universidade Federal do Rio Grande do Sul. Brasil. E-mail vladimir.lavayen@ufrgs.br

Recebido em: 28/08/2020; Aceito em: 22/04/2021

https://doi.org/10.5335/rbecm.v4i2.11599

http://creativecommons.org/licenses/by-nc-nd/4.0

ISSN: 2595-7376 


\section{Introdução}

O conceito de estequiometria (do grego stoicheion = elemento e metria = medida) é a base para o entendimento da diferença entre a química quantitativa e a qualitativa. (MENDES; SANTANA; JÚNIOR, 2017). As relações estequiométricas são um dos tópicos mais difíceis de serem compreendidos pelos alunos. A dificuldade dos alunos normalmente está relacionada à forma como o conteúdo é abordado, com enfoque no aspecto matemático em detrimento de uma interpretação química, levando a uma mecanização dos procedimentos para a solução de problemas envolvendo aspectos quantitativos dos fenômenos químicos. O desenvolvimento do cálculo estequiométrico engloba três linguagens: matemática (aritmética e proporção); física (unidades de medida); e química (simbologia, grandezas e equações químicas). (DA COSTA; DA TRINDADE SOUZA, 2013).

No domínio cognitivo, essas linguagens estão relacionadas com os três níveis representacionais da química (simbólico, submicroscópico e macroscópico), além do conhecimento matemático. Gabel (1999) classifica os sistemas em três níveis específicos: simbólico, macroscópico e submicroscópico. Esses níveis são assim detalhados: o nível macroscópico diz respeito às representações mentais adquiridas a partir da experiência sensorial direta, sendo construído mediante a informação proveniente dos sentidos; o submicroscópico está correlacionado às representações abstratas, com as entidades pequenas (ou seja, átomos, íons, moléculas); o nível simbólico, por sua vez, expressa os conceitos químicos a partir de fórmulas, equações, entre outros. (JOHNSTONE, 1991; GILBERT, TREAGUST, 2009).

No caso específico da estequiometria, as letras são utilizadas para representar elementos e suas fórmulas moleculares; os coeficientes estequiométricos indicam a proporção de moléculas na reação; e os índices numéricos, o número de átomos de uma espécie em particular. $\mathrm{O}$ vocabulário químico associado a essas operações parece dificultar a aplicação das habilidades matemáticas dos alunos aos fenômenos químicos. (HAIM et al., 2003). A complexidade do vocabulário associada a problemas estequiométricos pode impedir que os estudantes usem operações matemáticas simples para resolvê-los.

Alguns pontos críticos na resolução desses cálculos são o entendimento de como as reações funcionam e, particularmente, a diferença entre condições reacionais estequiométricas, conhecidas na literatura como "estequiometrias de reagente limitante". 
(LE MAIRE et al., 2018). Podem-se mencionar, também, nos casos avançados, os compostos que não contêm moléculas discretas com composição atômica variável, os quais são conhecidos como compostos não estequiométricos. (MYERS; MAHAN, 1995).

Kalantar (1985) declara que, durante anos, seus alunos tiveram problemas de estequiometria envolvendo um reagente limitante, especialmente em casos em que a estequiometria da reação não era de 1:1, mesmo compreendendo que as quantidades estequiométricas de reagentes eram exceção, e não regra, e apesar dos tratamentos, geralmente claros, apresentados nos textos. Muitos dos erros comuns cometidos pelos alunos, ao determinar a quantidade de produto formado, envolvem: reconhecer o reagente que está em menor quantidade como sendo o reagente limitante; calcular a quantidade de produto como sendo o somatório das quantidades de reagentes; não considerar os rearranjos dos átomos nas moléculas durante a reação química; resolver o problema diretamente com quantidade em gramas, não convertendo para número de mols; não perceber que haverá algum excesso de reagente no final da reação; e não atribuir os coeficientes aos reagentes descritos. (SOSTARECZ; SOSTARECZ, 2012).

A estequiometria de reações é um ponto crítico, sobretudo quando se trata de entender como funcionam as reações e a diferença entre condições de reação estequiométrica e não estequiométrica, também conhecida como "estequiometria do reagente limitante”. (LE MARIE et al., 2018). Isso ocorre devido ao fato de que os problemas com reagente limitante não são apenas abstratos, mas também matemáticos. (STEINER, 1986).

Um método para favorecer o domínio dos alunos a respeito de condições estequiométricas e não estequiométricas são as “analogias ilustradas”. (LE MAIRE et al., 2018). Segundo Gafoor e Shilna (2012), o uso de analogias é recomendado porque fornece uma ponte entre um conceito desconhecido e o conhecimento que os alunos têm, o que os motiva e facilita a visualização de conceitos abstratos por comparações com objetos concretos. As ideias desses autores vão ao encontro do predito pela Teoria da Aprendizagem Significativa, de David Ausubel.

\section{A aprendizagem significativa e a UEPS}

A Teoria da Aprendizagem Significativa, proposta por David Ausubel, objetiva compreender como o conhecimento é adquirido e a nossa estrutura cognitiva é construída. (AUSUBEL, 2003). Na perspectiva do autor, a aprendizagem é um pro- 
cesso por meio do qual uma nova informação (as ideias expressas simbolicamente) interage de forma substantiva (não literal) e não arbitrária com aquilo que o sujeito já sabe. (MOREIRA, 2013). A interação de forma literal está relacionada com a aprendizagem mecânica, uma vez que esse tipo de interação, em geral, limita-se a reproduzir o conhecimento de maneira idêntica à que foi apresentada ao aprendiz.

A aprendizagem mecânica fundamenta-se na memorização de novas ideias sem estabelecer conexões. Em vista disso, trata-se de um aprendizado com menores chances de permanecer na estrutura cognitiva do aluno a longo prazo. Contudo, se há o estabelecimento de conexões entre a nova informação e o conhecimento prévio, tem-se como resultado a construção de significados pessoais para essa informação. A construção de significados pessoais é elaborada de forma "não literal"; desse modo, se caracteriza como uma aprendizagem significativa. (TAVARES, 2004). A interação não arbitrária não deve ocorrer com qualquer ideia prévia, mas, sim, com algum conhecimento, especificamente relevante, já existente na estrutura cognitiva do aprendiz. Esse conhecimento prévio, isto é, aquilo que o sujeito já sabe, é o que Ausubel chamava de subsunçor ou ideia-âncora.

Em outras palavras, a nova informação ancora-se em conceitos ou proposições relevantes já presentes na estrutura cognitiva. A importância dos subsunçores no processo de aprendizagem é enfatizada por Ausubel, Novak e Hanesian (1980). Segundo os autores, se tivessem que reduzir a um princípio único toda a psicologia educacional, esse princípio seria: "O fator isolado mais importante que influencia a aprendizagem é aquilo que o aprendiz já conhece. Descubra o que ele sabe e baseie nisso os seus ensinamentos". (AUSUBEL; NOVAK; HANESIAN, 1980, p. 137).

Quando o aluno não dispõe de subsunçores adequados para a ancoragem da nova informação, pode-se recorrer aos organizadores prévios. Organizador prévio é um recurso que deve preceder o material de aprendizagem, ou seja, deve ser utilizado antes da abordagem do conhecimento científico a ser desenvolvido. Esses organizadores devem ter um nível mais alto de abstração, generalidade e inclusividade em relação a este. Além de suprir a deficiência de subsunçores, os organizadores prévios podem ser usados para auxiliar os alunos a perceberem a relacionabilidade entre os novos conhecimentos apresentados e os seus conhecimentos pré-existentes. (MOREIRA, 2013).

Assim, a aprendizagem significativa é considerada uma ampliação na estrutura cognitiva que opera como âncora para novos conceitos e ideias, estabelecendo rela- 
ções entre as ideias e organizando-as hierarquicamente. Para que a aprendizagem ocorra de maneira significativa, é fundamental que o material de aprendizagem seja potencialmente significativo (material relacionável à estrutura cognitiva do sujeito) e que o aprendiz tenha predisposição para aprender (dispor de conhecimentos prévios/subsunçores necessários). (MOREIRA, 2013).

O professor tem o papel de facilitar a construção dessas relações lógicas, utilizando os princípios de diferenciação progressiva e reconciliação integrativa, que são pressupostos dessa teoria. Na diferenciação progressiva, apresentam-se os conceitos mais gerais, os quais são discriminados progressivamente em conceitos mais específicos. A reconciliação integrativa consiste na construção e reconstrução das relações conceituais, visto que novos conceitos foram incorporados à estrutura cognitiva e precisam ser reorganizados (KONFLANZ et al, 2020).

Visando a facilitar essa relação lógica, Moreira (2011) propõe a criação de materiais potencialmente significativos, com uma estrutura organizada, que façam sentido ao público para o qual se pretende desenvolver determinado conceito e contribuam para uma aprendizagem com qualidade, se diferenciando da aprendizagem mecânica. As denominadas unidades de ensino potencialmente significativas (UEPSs) são sequências de ensino alicerçadas em teorias de aprendizagem que visam à aprendizagem significativa, em contraposição à aprendizagem puramente mecânica. Esse tipo de proposta didática tem como argumento principal a contribuição para uma modificação no ensino, que, ao longo das últimas décadas, tem sido pautado na memorização de conteúdo, proporcionando apenas a aprendizagem mecânica. (MOREIRA, 2011).

Sob essa ótica, o papel do professor é apresentar situações-problema, cuidadosamente selecionadas, para organizar o ensino, mediando a captação de significados da parte do aluno. Depois de definido o objetivo da sequência didática e identificados os aspectos declarativos e procedimentais sobre o tópico a ser abordado, Moreira (2011) recomenda que a UEPS seja organizada de acordo com aspectos sequenciais, que são, resumidamente, os seguintes:

Situação inicial: Nessa etapa, devem-se propor situações para que o aluno externalize seus conhecimentos prévios em relação ao conteúdo. Discussões, questionários e mapas conceituais são exemplos de atividades para essa fase.

Situação-problema inicial: De posse dos conhecimentos prévios dos alunos, deve-se propor uma situação-problema de nível introdutório, envolvendo o tópico a ser 
estudado, buscando dar um sentido à temática. Podem ser sugeridas situações por meio de simulações, demonstrações, vídeos, problemas cotidianos, matérias midiáticas, exercícios clássicos, desde que tenham um nível acessível e problematizador. A organização de uma situação-problema inicial é imprescindível para a aprendizagem significativa. São as situações-problema que dão sentido aos novos conhecimentos.

Aprofundamento do conteúdo: $\mathrm{O}$ conhecimento deve ser apresentado a partir da diferenciação progressiva, partindo-se de aspectos mais gerais, com uma visão integrada dos elementos relevantes à matéria de ensino para a exemplificação de pormenores específicos. Devem-se valorizar atividades colaborativas após a exposição do conteúdo, como práticas em grupos e apresentações. Conceitos estruturantes devem ser apresentados em níveis crescentes de complexidade, sempre destacando semelhanças e diferenças entre os exemplos, em uma reconciliação integrativa.

Nova situação-problema: A nova situação-problema deve propor questionamentos com um nível de complexidade maior, evidenciando as correspondências e contradições entre os conceitos. As atividades devem valorizar ações colaborativas entre os alunos, proporcionando maior interação e negociação de significados, sempre com a mediação do professor. Podem-se propor tarefas como resolução de problemas, construção de mapas conceituais, experimentos, elaboração de projetos, etc.

Aula integradora final: Abordam-se, aqui, as características mais relevantes do conteúdo em questão, dando continuidade ao método de diferenciação progressiva a partir de uma ótica integradora, buscando a reconciliação integrativa entre os conceitos.

Avaliação da aprendizagem na UEPS: A avaliação, nessa perspectiva, deve buscar evidências de compreensão de significados e capacidade de transferência do conhecimento para diferentes situações. Inclui a avaliação formativa (situações, tarefas em grupo, registros do professor), que visa a avaliar o progresso ao longo do processo de aprendizagem, e a avaliação somativa individual, na qual deverão ser propostas questões/situações, em geral, no formato de provas que demandem a compreensão e evidenciem a captação de significados. (MOREIRA, 2011). A avaliação deverá estar baseada, em pé de igualdade, tanto na formativa quanto na somativa individual.

Avaliação da UEPS: A fim de aprimorar e/ou validar as estratégias de ensino, além da evolução conceitual dos alunos, deve ser considerada uma avaliação con- 
junta sobre a UEPS. Tal avaliação pode se dar por meio de uma roda de conversa ou mesmo de um questionário avaliativo.

\section{A proposta de unidade de ensino potencialmente significativa}

Objetivando contribuir com o ensino de estequiometria, elaborou-se uma sequência de aulas baseadas nos pressupostos da aprendizagem significativa, utilizando como organizador prévio a relação com ingredientes culinários (Quadro 1). A sequência proposta será descrita a seguir, de acordo com Moreira (2013), tendo como objetivo ensinar os conceitos e cálculos relacionados à estequiometria de reações, usando exemplos de receitas culinárias como organizadores prévios.

Quadro 1: Organização da unidade de ensino

\begin{tabular}{|l|l|l|}
\hline Sequência & \multicolumn{1}{|c|}{ Etapas da UEPS } & \multicolumn{1}{|c|}{ Estratégias utilizadas } \\
\hline Aula 1 & $\begin{array}{l}\text { Situação inicial; } \\
\text { Situações-problema iniciais; } \\
\text { Aprofundamento do conteúdo. }\end{array}$ & $\begin{array}{l}\text { Mapa mental; } \\
\text { Resolução da situação-problema inicial; } \\
\text { Aula expositiva com uso de organizador prévio e reso- } \\
\text { lução de cálculos. }\end{array}$ \\
\hline Aula 2 & $\begin{array}{l}\text { Nova situação-problema; } \\
\text { Aprofundamento do conteúdo. }\end{array}$ & $\begin{array}{l}\text { Aula expositivo-dialogada com uso de organizador pré- } \\
\text { vio, simulador computacional e resolução de cálculos. }\end{array}$ \\
\hline Aula 3 & Aprofundamento do conteúdo. & Aula expositiva e resolução de cálculos. \\
\hline Aula 4 & Aprofundamento do conteúdo. & $\begin{array}{l}\text { Aula expositivo-dialogada com uso de organizador } \\
\text { prévio (atividade prática) e resolução de cálculos. }\end{array}$ \\
\hline Aula 5 & Aprofundamento do conteúdo. & Resolução de cálculos. \\
\hline Aula 6 & $\begin{array}{l}\text { Avaliação formativa; } \\
\text { Avaliação da UEPS. }\end{array}$ & $\begin{array}{l}\text { Mapa conceitual final; } \\
\text { Resolução de questão-desafio; } \\
\text { Aplicação de questionário com questões abertas e } \\
\text { fechadas para avaliação da UEPS. }\end{array}$ \\
\hline Aula 7 & Avaliação somativa individual. & Aplicação de prova. \\
\hline Aula 8 & Aula final. & $\begin{array}{l}\text { Resolução de dúvidas referentes às questões da ativi- } \\
\text { dade avaliativa. }\end{array}$ \\
\hline
\end{tabular}

Fonte: Elaborado pelos autores (2020).

Situação inicial: Com o intuito de instigar os alunos a externalizarem seus conhecimentos prévios, sugere-se uma atividade colaborativa em pequenos grupos (dois a quatro participantes) para a construção de um mapa mental (Figura 1), partindo da palavra central: "estequiometria". Esse mapa mental pode ser apresentado 
ao grande grupo e servir como base para a avaliação formativa. Para auxiliar os alunos nessa construção, podem ser feitas perguntas como: O que é estequiometria? O que o preparo de uma receita tem a ver com química? Também podem ser lançadas palavras-chave como: proporção, unidades de medida, conversão de unidades, grandezas, equação química, coeficientes estequiométricos.

Figura 1: Atividade colaborativa: mapa mental sobre estequiometria

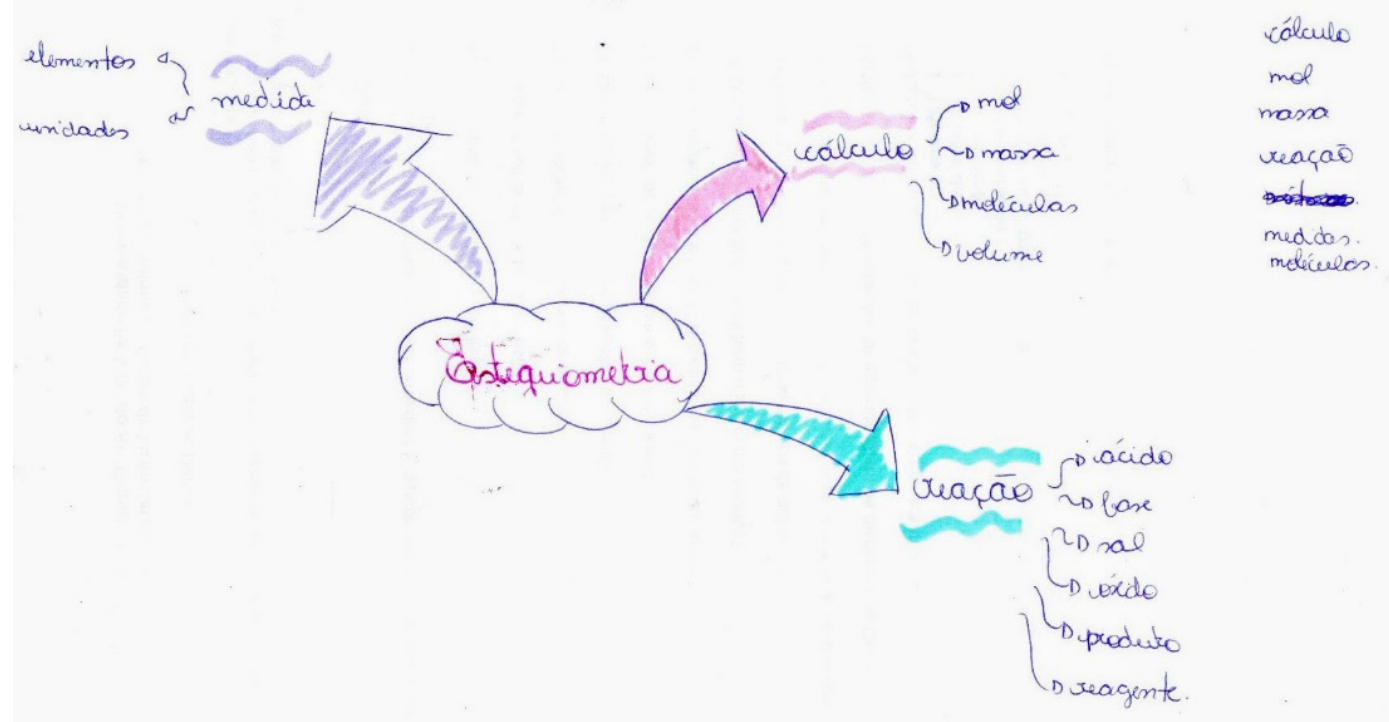

Fonte: Elaborada pelos autores (2020).

Situações-problema iniciais: Para o ensino de estequiometria, optou-se por utilizar como situações-problema iniciais questões do cotidiano, fazendo uso da relação com ingredientes culinários. Os alunos são convidados a resolver problemas a partir de receitas culinárias fornecidas pelo professor. Propõe-se que as questões contemplem, em nível introdutório, relações que serão importantes no desenvolvimento do conteúdo (como proporções e unidades de medida) e que os alunos possam modelá-las mentalmente. As Figuras 2 e 3 contém exemplos que podem ser utilizados. 
Figura 2: Receitas utilizadas

Receita de bolo 1 :

- 4 unidades de gema de ovo

- 4 unidades de clara de ovo batidas em neve

- 2 xícaras (chá) de farinha de trigo

- 1 xícara (chá) de açúcar

- 1 colher (sopa) de fermento químico em pó

- 1 xícara (chá) de água Porção: $540 \mathrm{~g}$
Receita de bolo 2:

- 2 xícaras (chá) de açúcar

- 3 xícaras (chá) de farinha de trigo

- 4 colheres (sopa) de margarina

- 3 ovos

- 1 e $1 / 2$ xícara (chá) de leite

- 1 colher (sopa) bem cheia de fermento em pó

Fonte: Elaborada pelos autores (2020).

Figura 3: Situações para cálculo com ingredientes culinários

Questões para responder (apresente os cálculos utilizados e/ou explique como chegou à resposta da questão):

1) Se você tiver em casa uma quantidade de açúcar equivalente a duas xícaras de chá, poderá fazer um bolo de quantos gramas usando a receita 1? Por quê? $\mathrm{E}$, nesse caso, precisará de quais quantidades dos demais ingredientes?

2) Suponha que você quer fazer o bolo da receita 2 utilizando os 5 ovos que estão na sua geladeira. Quantas xícaras de açúcar terá que utilizar? Sabendo que uma xícara de chá corresponde ao volume de $240 \mathrm{~mL}$, qual o volume de leite será necessário?

3) Você não encontrou nenhuma xícara de chá em sua cozinha mas, por outro lado, possui uma balança de cozinha e um copo medidor. Se, ao pesar a farinha, você encontrou uma massa de $500 \mathrm{~g}$ e deseja utilizar toda essa quantidade para fazer a receita 2 , qual volume de leite terá que medir para adicionar à massa do bolo?

Dado: 1 xícara (chá) = $120 \mathrm{~g}$ de farinha ou $240 \mathrm{~mL}$ de leite

4) Antes de começar a fazer a receita 2 , você conferiu se teria ingredientes o suficiente para fazer mais que um bolo. Verificou que ainda tinha 6 xícaras de farinha de trigo e uma dúzia de ovos. Tendo quantidade suficiente dos demais ingredientes, quantos bolos poderá fazer? Qual destes dois ingredientes sobrará?

Fonte: Elaborada pelos autores (2020).

Aprofundamento do conteúdo: Uma aula expositivo-dialogada pode ser utilizada como estratégia para começar a ensinar, de fato, o tópico em pauta. A partir dos resultados da etapa anterior, é possível verificar se os alunos têm em sua estrutura cognitiva os subsunçores adequados para o desenvolvimento do conteúdo ou identificar a necessidade de empregar organizadores prévios.

Um exemplo de receita de torrada (Figura 4) escrita na forma de uma equação química, é uma alternativa simples de organizador prévio que pode preceder a 
apresentação dos aspectos gerais do conteúdo a ser desenvolvido (Lei de Lavoisier ou Lei da Conservação da Matéria, Lei de Proust ou Lei das Proporções Constantes, relações molares, relações mol/massa, relações massa/massa, entre outras, aplicadas ao cálculo estequiométrico), buscando explicitar a relacionabilidade entre o conhecimento do cotidiano do aluno e o conhecimento novo.

Figura 4: Uso de organizadores prévios para relações não estequiométricas

\section{Receita 1: torrada}

$2 \mathrm{P}+1 \mathrm{Pr}+1 \mathrm{Qu} \rightarrow 1 \mathrm{P}_{2} \mathrm{PrQu}$

$\mathrm{P}=$ pão; $\mathrm{Pr}=$ presunto; $\mathrm{Qu}=$ queijo
Receita 2: omeletes

6 ovos +1 xícara de queijo ralado $\rightarrow$ 2 omeletes

Questão 1. Se, para fazer a receita 1, você tiver 10 fatias de pão, 4 fatias de presunto e 6 fatias de queijo:

A) Qual é a quantidade máxima de torrada que poderá fazer com estes ingredientes?

B) Qual é o ingrediente limitante?

C) Quais são os ingredientes em excesso? Quanto sobrará de cada um deles depois que fizer as torradas?

Questão 2. Se, para fazer a receita 2 , tiver 5 ovos e $1 / 2$ xícara de queijo ralado:

A) Qual é a quantidade máxima de omelete que poderá fazer com estes ingredientes?

B) Qual é o ingrediente limitante?

C) Qual é o ingrediente em excesso? Quanto sobrará dele após fazer a receita?

Questão 3. Sabendo que 6 ovos $=1200 \mathrm{~g} \mathrm{e} 1$ xícara de queijo ralado $=50 \mathrm{~g}$, responda aos itens $\mathrm{A}, \mathrm{B}$ e $\mathrm{C}$ da questão 2 , desta vez considerando que você possui $600 \mathrm{~g}$ de ovos e $30 \mathrm{~g}$ de queijo ralado.

Fonte: Elaborada pelos autores (2020).

Nova situação-problema: Como as novas situações-problema devem ser propostas em níveis crescentes de complexidade, devem ser abordadas, nesse momento, situações em que os reagentes não estão em relações estequiométricas, em que um deles pode se consumir completamente antes, destacando semelhanças e diferenças relativamente às situações e aos exemplos nos quais esteja presente o uso de relações algébricas simples, a fim de promover a reconciliação integrativa. Segundo Moreira (2013), devem-se explorar relações entre ideias, conceitos e proposições, bem como apontar similaridades e diferenças importantes, reconciliando discrepâncias reais ou aparentes. 
Indica-se a realização de atividade com reagentes, produtos e excesso do simulador interativo gratuito do Portal PhET, que, dependendo dos recursos à disposição, pode ser efetuada em laboratório de informática ou em aparelho celular com acesso à Internet. Nessa tarefa, os alunos devem ter à disposição diferentes quantidades de reagentes/ingredientes e, com o auxílio da ilustração, pode-se verificar qual a quantidade máxima de produto a ser obtida, qual(is) reagente(s)/ingrediente(s) está(ão) em excesso e qual deles está limitando a formação de produto, atentando-se ao rearranjo dos átomos nas moléculas ocorrido na reação química.

Convém convidar os alunos a jogarem os diferentes níveis do jogo disponíveis no simulador, desafiando-os a utilizar também as opções de ocultar moléculas e ocultar os números. Na Figura 5, tem-se um exemplo do uso dos ingredientes culinários, empregados nessa proposta como organizadores prévios. O uso de analogias ilustradas é recomendado por Gafoor e Shilna (2012), pois faz uma ponte entre um conceito abstrato e os conhecimentos prévios dos alunos.

Figura 5: Atividade com simulação para reagentes em excesso e limitantes

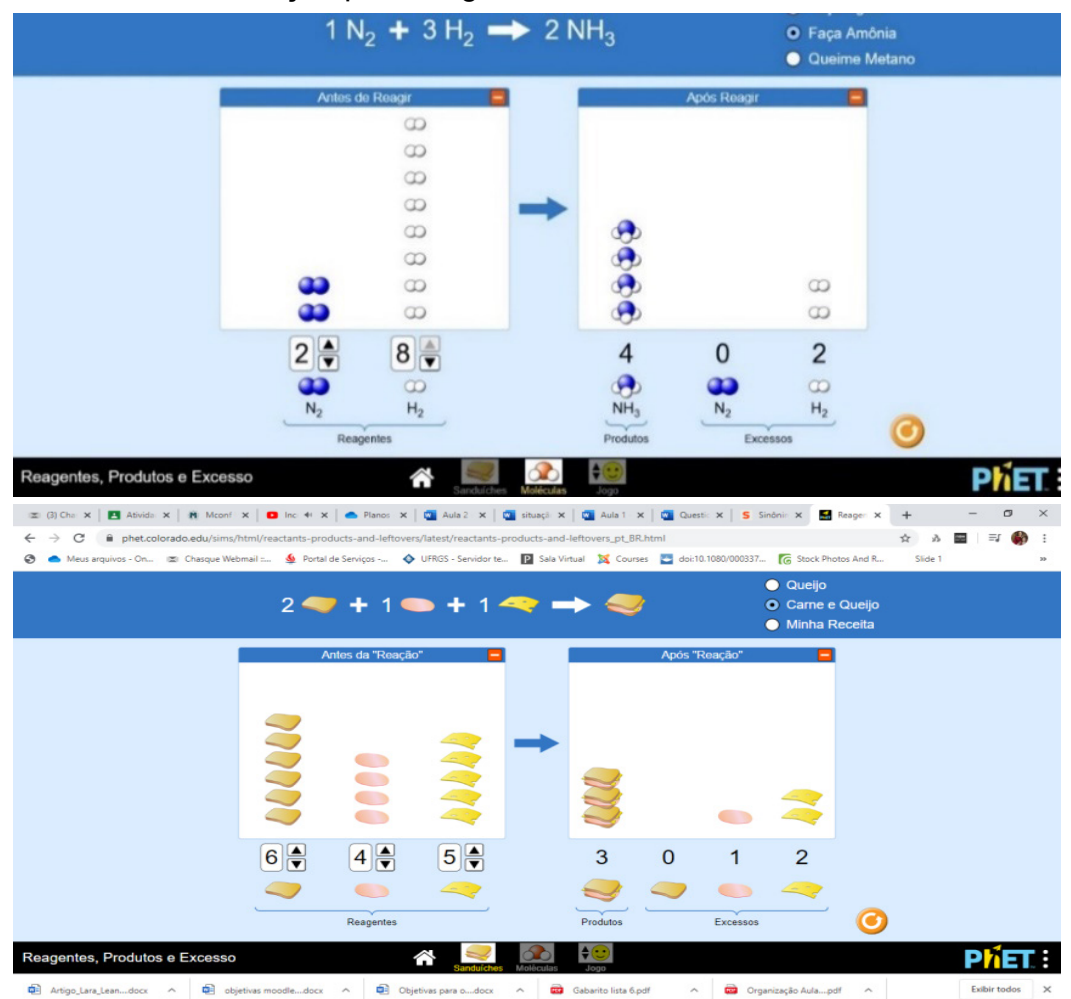

Fonte: Elaborada pelos autores (2020). 
Aprofundamento do conteúdo: Sugere-se novamente uma aula expositivo-dialogada, iniciando com exemplos culinários simples - como receita de sanduíche e de omeletes -, escritos na forma de equações químicas, tendo a função de atuar como organizadores prévios, dessa vez para o entendimento dos conceitos de reagente limitante e de reagente em excesso. Após a explanação dos conteúdos, é conveniente que os alunos tenham tempo em aula para resolver exercícios que envolvam situações de reações em condições estequiométricas, solicitando o auxílio do professor se necessário. Ainda dentro do aprofundamento do conteúdo, podem ser trabalhadas questões envolvendo pureza de reagentes e rendimento das reações. Anteriormente à introdução deste último tópico, indica-se a realização da atividade prática, conforme a Figura 5, podendo atuar como um organizador prévio, usando novamente um exemplo culinário como analogia para um conceito químico.

Figura 6: Atividade prática sobre rendimento de reações

Atividade: Solicitar aos alunos que separem 50 grãos de milho de pipoca e os insiram pipoqueira elétrica. Depois que a pipoca terminar de estourar, deverão contar a quantidade de grãos que estourou e que não estourou para em seguida:

1. Calcular o rendimento percentual do "processo".

2. Considerando este rendimento, calcular a quantidade de milho que deveria ser colocada na pipoqueira para ter 50 grãos de pipoca estourada.

Fonte: Elaborada pelos autores (2020).

Avaliação formativa: Indica-se a construção de um mapa conceitual (Figura 7) como atividade avaliativa. É interessante que sejam mantidos os mesmos grupos da construção do mapa mental (situação inicial) para que estes possam ser comparados na busca de evidências de aprendizagem significativa. Outra atividade que pode compor a avaliação formativa é a resolução de uma questão-desafio, tendo as características de uma situação-problema, em que os diversos aspectos abordados no aprofundamento do conteúdo sejam aplicados de forma contextualizada. 
Figura 7: Atividade colaborativa: mapa conceitual de estequiometria

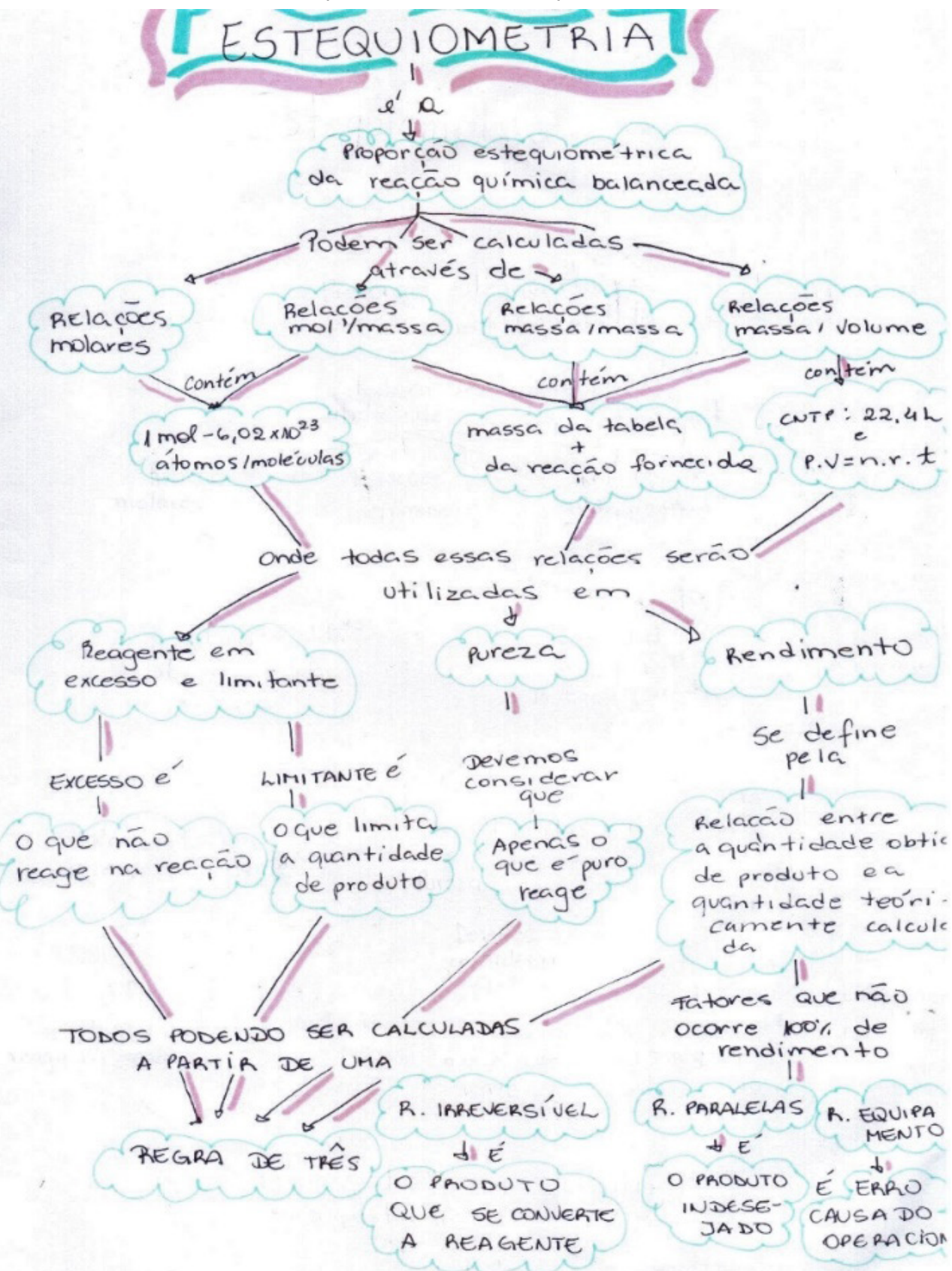

Fonte: Elaborada pelos autores (2020). 
Avaliação da UEPS:A fim de aprimorar e/ou validar a estratégia utilizada para o ensino de estequiometria, além das avaliações formativas e somativas que visam a identificar a evolução conceitual dos alunos, buscando indícios de aprendizagem significativa, é importante que os estudantes avaliem a unidade de ensino proposta. Essa avaliação pode se dar por meio de uma roda de conversa, ou mesmo um questionário avaliativo com questões fechadas do tipo escala Likert de cinco pontos e questões abertas.

Avaliação somativa individual: Para avaliar o alcance de determinados objetivos de aprendizagem ao final da aplicação da UEPS, comumente realiza-se uma avaliação baseada em provas. A sugestão é que sejam utilizadas tanto questões com ingredientes culinários quanto questões de estequiometria (Figura 8).

Figura 8: Exemplos de questões para avaliação somativa individual

9. Pesando em uma balança de cozinha os ingredientes disponíveis, você verificou que possui $156 \mathrm{~g}$ de ovos, $250 \mathrm{~g}$ de leite, além de 2 latas de leite condensado. A partir destes ingredientes, será possível fazer um pudim de até quantos gramas, seguindo a receita abaixo? $(0,8 \mathrm{pt})$

Receita de pudim (890g):

1 lata de leite condensado

1 xícara de leite $(300 \mathrm{~g})$

3 ovos (195 g)

10. O sulfato de bário é usado como contraste em exames radiológicos e pode ser obtido pela reação de cloreto de bário com ácido sulfúrico. Se forem adicionados $150 \mathrm{~g}$ de cloreto de bário a uma solução contendo $80 \mathrm{~g}$ de ácido sulfúrico, qual será a massa de sulfato de bário produzida? $(0,8 \mathrm{pt})$

Fonte: Elaborada pelos autores (2020).

Aula final: Resolução de dúvidas referentes às questões da atividade avaliativa.

\section{Considerações finais}

O debate sobre ensinar conceitos científicos de forma contextualizada não é novidade no ensino de química, mas se configura como um desafio em sala de aula. Estabelecer relações diretas com o dia a dia, o contexto ou questões atuais com as quais os alunos têm contato é uma estratégia para dar sentido aos conceitos abordados e aproximar os estudantes da ciência. 
Dessa forma, o uso das UEPS pode ser considerado uma alternativa prática e viável para ser implementada em sala de aula, uma vez que propõe uma organização lógica e sequencial das atividades de ensino, com o emprego de situações em ordem crescente de complexidade e com avaliação ao longo de sua implementação. Essas são estratégias fundamentais para o processo de conceitualização e que irão contribuir para que o aluno atribua sentido a esse novo conceito, promovendo uma aprendizagem com significado.

Em outras palavras, trata-se de uma organização didática com vistas a uma aprendizagem que não é limitada à memorização e à reprodução em uma avaliação, pois objetiva a compreensão, a capacidade de explicar e aplicar o conhecimento em novas e diferentes situações de forma processual. Espera-se que a UEPS aqui apresentada e detalhada incentive o desenvolvimento e a implementação em sala de aula de outras unidades de ensino potencialmente facilitadoras da aprendizagem significativa, principalmente para os tópicos específicos que são considerados mais desafiadores tanto pelos alunos quanto pelos professores. Ademais, a UEPS aplicada estará sendo analisada como parte de um trabalho de conclusão de curso de Licenciatura em Química.

\section{The use of previous organizers for teaching stoichiometry: potentially significant teaching unit proposal}

\section{Abstract}

This work shows a didactic proposal for the teaching of stoichiometry based on the theory of significant learning and organized in the format of a potentially significant teaching unit. The class sequence format aims to promote quality learning, which differs from machine learning. Taking into account that the previous knowledge of the students strongly influences learning. Situations and problems involving the relationship with ingredients and culinary recipes are used here to bridge new knowledge. The justification for developing this teaching strategy is because stoichiometry is identified as a challenging subject, due to the need for the use and interpretation of chemical, physical, and mathematical languages. As a result, we hope that this unit will contribute to a better understanding of stoichiometry concepts and promote the use of learning materials that are potentially significant and demonstrate the construction of logical relationships with scientific knowledge and distancing of the chemistry teaching based solely on memorizing contents.

Keywords: chemistry teaching; meaningful learning; previous knowledge; stoichiometric calculation; limiting reagent 
AUSUBEL, David. P. Aquisição e retenção de conhecimentos: uma perspectiva cognitiva. Lisboa: Editora Plátano, 2003.

AUSUBEL, David. P.; NOVAK, Joseph. D.; HANESIAN, Helen. Psicologia Educacional. 2. ed. Rio de Janeiro: Editora Interamericana,1980.

DA COSTA, Ana Alice Farias; DA TRINDADE SOUZA, Jorge Raimundo. Obstáculos no processo de ensino e de aprendizagem de cálculo estequiométrico. Amazônia: Revista de Educação em Ciências e Matemáticas, v. 10, n. 19, p. 106-116, 2013.

GABEL,Dorothy. Use of the particle nature of matter in developing conceptual understanding. Journal of Chemical Education, v. 70, n.3, p.193-194, 1993.

GAFOOR, K. Abdul.; SHILNA, V. Chemistry instruction through analogies. Presented at the National Seminar on Emerging Trends in Education at the Department of Education; University of Calicut: Kerala, India, 11-12 nov. 2012.

GILBERT, John. K.; TREAGUST, David. F. Multiple representations in chemical education. Dordrecht: Springer, 2009. 390p.

HAIM, Liliana et al. Learning stoichiometry with hamburger sandwiches. Journal of Chemical Education, v. 80, n. 9, p. 1.021, 2003.

JOHNSTONE, Alex. H. Why science difficult to learn? Things are seldom what they seem. Journal Computer Assisted Learning, Hoboken, v .7, n. 2, p. 75-83, 1991.

KALANTAR, Alfred. H. Limiting reagent problems made simple for students. Journal of Chemical Education, v. 62, n. 2, p. 106, 1985.

KONFLANZ, Gabriel. Muller et al. Unidade de ensino potencialmente significativa mediada pelas Tecnologias de Informação e Comunicação para o ensino de séries de Fourier. Revista Brasileira de Ensino de Ciências e Matemática, v. 2, n. 2, 2019.

LE MAIRE, Nathalie V. et al. Clash of chemists: a gamified blog to master the concept of limiting reagent stoichiometry. Journal of Chemical Education, v. 95, n. 3, p. 410-415, 2018.

MENDES, Abinadabis; SANTANA, Genilson; JÚNIOR, Erasmo Pessoa. O uso do software PhEt como ferramenta para o ensino de balanceamento de reação química. Revista Areté/Revista Amazônica de Ensino de Ciências, v. 8, n. 16, p. 52-60, 2017.

MOREIRA, Marco Antonio. Aprendizagem significativa, organizadores prévios, mapas conceituais, diagramas $\mathrm{V}$ e unidades de ensino potencialmente significativas. Material de apoio para o curso Aprendizagem Significativa no Ensino Superior: Teorias e Estratégias Facilitadoras. Pontifícia Universidade Católica do Paraná, 2012-2013.

MOREIRA, Marco Antonio. O que é afinal Aprendizagem Significativa? Aula Inaugural do Programa de Pós-Graduação em Ensino de Ciências Naturais, Instituto de Física, Universidade Federal do Mato Grosso, Cuiabá, MT, 23 de abril de 2010. Instituto de Física, UFRGS. Disponível em: <http://www.if.ufrgs.br/ moreira/oqueeafinal.pdf>. Acesso em: 02 ago. 2019.

MOREIRA, Marco Antonio. Unidades de enseñanza potencialmente significativas - UEPS. Potentially meaningful teaching units PMTU. Aprendizagem Significativa em Revista, v. 1, 
n. 2, p. 43-63, 2011. Porto Alegre: Instituto de Física da UFRGS, 2011. Disponível em: http://www. if.ufrgs.br/asr/artigos/Artigo_ID10/v1_n2_a2011.pdf. Acesso em: 20 jun. 2019.

MYERS, Rollie J.; MAHAN, Bruce M. Química: um curso universitário. São Paulo: Blucher, 1995.

SOSTARECZ, Michael C.; SOSTARECZ, Audra Goach. A conceptual approach to limiting-reagent problems. Journal of Chemical Education, v. 89, n. 9, p. 1.148-1.151, 2012.

STEINER, Richard P. Teaching stoichiometry. Journal of Chemical Education, v. 63, n. 12, p. 1.048, 1986.

TAVARES, Romero. Aprendizagem significativa. Revista Conceitos, v. 55, n. 10, 2004. 